\title{
Retardation of non-thermal photon light curves from flaring blazars
}

\section{Synchrotron radiation}

\author{
B. Eichmann ${ }^{1,2}$, R. Schlickeiser ${ }^{1}$, and W. Rhode ${ }^{2}$ \\ 1 Institut für Theoretische Physik, Lehrstuhl IV: Weltraum- und Astrophysik, Ruhr-Universität Bochum, 44780 Bochum, Germany \\ e-mail: rsch@tp4.ruhr-uni-bochum.de \\ 2 Experimentelle Physik V, Technische Universität Dortmund, 44221 Dortmund, Germany
}

Received 23 July 2009 / Accepted 28 November 2009

\section{ABSTRACT}

\begin{abstract}
An analytical model is presented which describes the intrinsic synchrotron intensity emitted by a spherical plasmoid volume of radius $R$ in the jet of an active galactic nucleus (AGN). Analytical results for the emergent synchrotron intensity could be achieved by using a monochromatic approximation for the synchrotron power. The synchrotron intensity is given by an infinite sum, reflecting the spatial eigenfunction distribution of the radiating electrons over the emission knot. The radiative transport of the generated synchrotron photons is simplified by the use of the escape probability concept which approximates the spatial photon diffusion caused by multiple Compton scatterings off thermal electrons in the knot. With these assumptions conclusions on the total duration of the synchrotron flare, its starting time and the cause for even shorter time variabilities are derived. The total flare duration at all photon energies $\epsilon$ equals the light travel time $2 R / c$. Shorter photon energy-dependent synchrotron intensity time variations are possible, which reflect the influence of the photon retardation and escape as well as the temporal and spatial dependences of the relativistic electron density distribution. The starting time of the synchrotron flare is delayed with respect to the injection time of ultrarelativistic electrons $t_{0}$ by a photon energy dependent time scale $\propto \epsilon^{-1 / 2}$, reflecting the necessary cooling time which relativistic electrons need in order to radiate at photon energies below the initial characteristic synchrotron photon energy $E_{0}$.
\end{abstract}

Key words. radiation mechanisms: non-thermal - galaxies: active - gamma rays: galaxies

\section{Introduction}

The recently launched AGILE and FERMI satellite mission together with new generation of ground-based air Cherenkov telescopes such as HESS, VERITAS and MAGIC provide unprecedented accurate coverage of our universe at $\mathrm{GeV}$ to $\mathrm{TeV}$ photon energies. The nearly all-sky field-of-view of FERMI and the much improved sensitivity of the air Cherenkov telescopes open up new opportunities to investigate especially powerful variable high-energy photon emitters such as active galactic nuclei (AGNs). The air Cherenkov telescopes have detected 27 AGNs as powerful variable high-energy photon emitters: apart from the radio galaxies $\mathrm{M} 87$, Cen $\mathrm{A}$ and $3 \mathrm{C} 66 \mathrm{~B}$ each of them belong to the blazar class which is characterized by rapid time variability at all wavelengths. These blazars often have established superluminal motion components at radio and $\mathrm{mm}$ frequencies. For strong enough flaring blazar sources at cosmological distances such as PKS 2155-304 (redshift $z=0.116$ ) (Aharonian et al. 2007) and Mrk $421(z=0.030)$ (Woo et al. 2005; Albert et al. 2007) the observed high fluxes now allow the determination of accurate photon spectra and light curves from $\mathrm{MeV}$ to $\mathrm{TeV}$ energies on time scales of minutes, often supplemented by dedicated time-correlated observations at lower radio to X-ray photon energies. These detailed observations allow us to perform crucial tests of our current understanding of the physical processes responsible for the generation of intense variable non-thermal radiation in these sources. In particular, it will be possible to determine the primary hadronic or leptonic nature of non-thermal radiation production in these sources, which is closely related to the question of the nature of cosmic particle accelerators, one of the eleven open fundamental problems of physics for the new millenium (Turner et al. 2003).

The combination of high observed luminosities with the observed short time variability in blazar flares indicates that the photon emission in blazars originates in relativistic jet knots that are beamed and Doppler-boosted towards the observer (e.g. Schlickeiser 1996). Superluminal expansions observed with VLBI (Piner \& Edwards 2004) provide evidence for moderate Doppler boosting factors in PKS 2155-304, although the $\mathrm{TeV}$ variability time scales require the presence of much larger Doppler factors in these jets. The properties of the observed non-thermal radiation from relativistic moving jet emission components are determined by intrinsic charged particle acceleration processes (intrinsic processes), environmental conditions causing high or low luminosity, external (to the jet) target photon fields, and Doppler boosting of beamed intensities and frequencies depending on the observer's viewing angle with respect to the direction of motion of the jet components (geometry influence).

The broadband continuum spectra of blazars are dominated by non-thermal emission and often consist of two distinct broad components. While there is considerable debate concerning the nature of the high energy spectral component (decay of neutral pions in hadronic emission models versus inverse Compton scattering of intrinsic (SSC) and external low-energy photons in leptonic emission models), it is agreed that the low-energy component is synchrotron radiation from highly relativistic electrons. The peak of the low-frequency flux density component is caused by the transition from optically thick to optically thin synchrotron radiation, and is located during the time of maximum 
$\gamma$-ray emission at infrared to optical frequencies. Only after considerable source expansion do these emission knots become optically thin at radio frequencies, where we can measure superluminal expansion speeds with VLBI techniques.

To model the multiwavelength flaring behaviour of blazars we consider a one-zone spherical (size $R=10^{15} R_{15} \mathrm{~cm}$ ), partly turbulent magnetised (field strength $B=1 b$ Gauss) emission knot, containing a non-relativistic plasma of electrons and ions (or positrons) of a density $N_{b}=10^{10} N_{10} \mathrm{~cm}^{-3}$, which moves with the relativistic speed $\beta^{*} c$ in a direction $\theta^{*}$ with respect to the observer, so that the Doppler factor $\delta=\left(1-\beta^{* 2}\right)^{1 / 2} /\left(1-\beta^{*} \cos \theta^{*}\right)$ applies to the Lorentz transformation of intensities and time scales from the coordinate system comoving with the emission knot to the observer's frame (see Appendix B for details). Multiple Compton scatterings of the generated synchrotron photons with optical depth $\tau=\sigma_{\mathrm{T}} N_{b} R=6.65 N_{10} R_{15}$, where $\sigma_{\mathrm{T}}$ is the Thomson cross section, then allow one to use the diffusion approximation for the spatial transport of synchrotron photons (Sunyaev \& Titarchuk 1980, 1985), implying a nearly isotropic synchrotron photon density distribution over the emission knot. The study represented here therefore is for an emission knot that is Thomson-thick from non-relativistic plasma. Such a high number density of non-relativistic background particles is needed in hadronic emission models for the jet emission (Pohl \& Schlickeiser 2000) to generate enough pions from inelastic hadron-hadron conditions. Moreover, an optical depth greater than unity for multiple Compton scattering of synchrotron photons in the knot justifies the diffusion approximation to the synchrotron photon transport considered below and the small anisotropy of the radiation field near the edge of the emission knot.

Note that below all physical quantities in the observer's frame are denoted by an asterisk. A chaotic magnetic field of a constant strength $B$ is adopted, which is randomly oriented on scales much larger than the gyroradii of the injected ultrarelativistic particles, so that classical angle-averaged synchrotron radiation formulae can be used (Crusius \& Schlickeiser 1986, 1988).

After accounting for the Doppler time dilatation, the intrinsic variability time scale $t_{\mathrm{var}}$ of radiation is caused by five different physical processes:

(1) the injection of the radiating relativistic particles in the emission knot due to acceleration or energisation processes;

(2) the spatial diffusion of the injected relativistic particles in the emission knot resulting from pitch-angle scattering in the partly turbulent and chaotic magnetic field;

(3) the radiative cooling of relativistic particles;

(4) the spatial escape of the generated non-thermal photons in the emission knot resulting from multiple Compton scattering off the cold electrons; and

(5) effects due to causality and retardation relating generated photon number densities inside the emission blob to the photon density at the edge $R$ of the emission blob from where they can escape freely.

Recently, Schlickeiser \& Röken (2008 - hereafter referred to as SR) have investigated the synchrotron and synchrotron-self Compton (SSC) flaring of blazars due to relativistic electrons, including processes (1) and (3). They assumed that at the moment $t=t_{0}$ monoenergetic ultrarelativistic electrons were instantaneously injected uniformly over the knot volume. Although other (than monoenergetic) injection energy spectra of ultrarelativistic electrons and other (than instantaneous) injection time profiles are physically plausible, the case of monoenergetic instantaneous injection is mathematically very convenient, as the solution of the time-dependent kinetic equation for the electrons for this case serves as Green's function for more complex injection energy spectra and time profiles, as long as the electron synchrotron cooling is linear, i.e. the magnetic field strength remains constant during the time evolution of the relativistic electrons (Schlickeiser \& Lerche 2007). The solutions for more complex injection energy spectra and time profiles $Q(E, t)$ are then simply obtained by quadratures involving the Green's function solution.

It is the purpose of the present paper to extend the SRanalysis by including the effects due to the spatial propagation of relativistic electrons (2) as well as photon propagation (4) and retardation (5), which arise due to the finite size of the emission knot. Our goal is to disentangle the variability effects caused by radiative cooling of the relativistic electrons from propagation and retardation effects. SR have found that at the optical, UV, $\mathrm{X}$-ray and $\gamma$-ray photon frequency bands optical thickness effects due to synchrotron self absorption are negligible. We will therefore restrict our discussion of photon propagation to the optically thin regime. Because of the complexity of the subject, we limit our analysis here to the variability of synchrotron radiation, whereas the variability of inverse Compton radiation (synchrotron-self-Compton and external Compton) will be addressed in future work.

The organisation of the paper is as follows: in Sects. 2 and 3 we discuss and solve the time-dependent evolution of the kinetic equations for the densities of radiating relativistic electrons $(n(r, \gamma, t))$ and for the non-thermal synchrotron photons $(M(r, \epsilon, t))$, neglecting absorption inside the emission knot. Because the spatial electron diffusion operator is of the SturmLiouville type, the solutions are obtained by expanding with respect to the self-adjoint diffusion eigenfunctions. The full radial dependence of the synchrotron photon density over the cloud is needed to account for the retardation effect. The emergent intrinsic synchrotron photon intensity is proportional to the synchrotron photon density $M(R, \epsilon, t)$ at the edge of the emission cloud, calculated in Sect. 4. For illustrative examples of the spatial variation of the ultrarelativistic electron injection rate, we calculate in Sect. 5 the resulting emergent synchrotron intensities.

\section{Kinetic equations of photons and electrons}

All physical quantities are calculated in a coordinate system comoving with the radiation source.

\subsection{Relativistic electrons}

The differential number density of relativistic electrons $n(r, \gamma, t)$ obeys the transport equation (with the relativistic electron Lorentz factor $\gamma \gg 1$ )

$\frac{\partial n}{\partial t}=\frac{1}{r^{2}} \frac{\partial}{\partial r}\left(r^{2} D_{0} \frac{\partial n}{\partial r}\right)+\frac{\partial}{\partial \gamma}[|\dot{\gamma}| n]+q_{1}(\gamma, t) q_{2}(r)$,

where the spatial diffusion is caused by rapid pitch-angle scattering in the partly turbulent chaotic magnetic field of the emission knot (Schlickeiser et al. 2002; Stockem \& Schlickeiser 2007). We adopt a constant diffusion coefficient

$D_{0}=c l_{0} / 3$ 
in terms of the mean scattering length $l_{0}$. We also adopt a separable source term of relativistic electrons $q_{1}(\gamma, t) q_{2}(r)$. Furthermore, the source interior should have an energy density of synchrotron radiation that is small compared to the energy density of the magnetic field $U_{B}$, so that the synchrotron-self Compton (SSC) energy losses are negligible compared to the synchrotron energy losses. For dominating SSC energy losses, the analysis becomes much more involved, as the SSC loss rate of a single electron depends on an energy integral of the electron density in the Thomson limit (see Schlickeiser 2009, for details). Our analysis therefore applies to those blazars where the low-frequency synchrotron component dominates over the high-frequency SSC component in the spectral energy distribution $v F_{v}$. The linear synchrotron energy loss rate is

$|\dot{\gamma}|=D_{\mathrm{s}} \gamma^{2}, \quad D_{\mathrm{s}}=\frac{4}{3} \frac{c \sigma_{\mathrm{T}}}{m_{\mathrm{e}} c^{2}} U_{B}=2.66 \times 10^{-14}\left[\frac{U_{B}}{m_{\mathrm{e}} c^{2}}\right] \mathrm{s}^{-1}$.

The particle transport Eq. (1) then reads

$\frac{\partial n}{\partial t}=\frac{c l_{0}}{3 r^{2}} \frac{\partial}{\partial r}\left(r^{2} \frac{\partial n}{\partial r}\right)+\frac{\partial}{\partial \gamma}[|\dot{\gamma}| n]+q_{1}(\gamma, t) q_{2}(r)$.

The spatial boundary conditions for electron propagation are

(a) finite density $n(0, \gamma, t)$ at the centre of the knot; and

(b) no particle flux from outside onto the boundary surface of the spherical knot:

$\left[D_{0} \frac{\partial n}{\partial r}+\frac{c}{2} n\right]_{r=R}=\left[\frac{c l_{0}}{3} \frac{\partial n}{\partial r}+\frac{c}{2} n\right]_{r=R}=0$,

implying

$\left[\frac{\partial n}{\partial r}+\frac{3}{2 l_{0}} n\right]_{r=R}=0$.

\subsection{Photon retardation}

The relativistic electrons emit photons isotropically at every point $\boldsymbol{r}=r\left(\sqrt{1-\mu^{2}} \cos \phi, \sqrt{1-\mu^{2}} \sin \phi, \mu\right)$ within the spherically-symmetric source where $r, \phi$ and $\mu=\cos \theta$ denote the photon's spherical coordinates. Let $\rho\left(r^{\prime}, t^{\prime}\right)$ denote the omnidirectional photon production rate per unit volume at a time $t^{\prime}$ and a distance $r^{\prime}$ from the centre of the source. If the source is optically thin, then the photon density at a time $t$, a distance $r$ and an energy $\epsilon$ is given by integrating over all emission points inside the source, generalising Gould's (1979) formula for nonstationary sources:

$M(r, \epsilon, t)=\frac{1}{c} \int_{-\infty}^{\infty} \mathrm{d} t^{\prime} \int \mathrm{d}^{3} r^{\prime} \frac{\rho\left(r^{\prime}, \epsilon, t^{\prime}\right)}{4 \pi s^{2}} \delta\left(t-t^{\prime}-\frac{s}{c}\right)$,

with the distance

$$
\begin{aligned}
s=\left|\boldsymbol{r}-\boldsymbol{r}^{\prime}\right|= & \left(\left(r^{2}+r^{\prime 2}-2 r r^{\prime}\left[\mu \mu^{\prime}+\sqrt{1-\mu^{2}}\right.\right.\right. \\
& \left.\left.\times \sqrt{1-\mu^{\prime 2}} \cos \left(\phi-\phi^{\prime}\right)\right]\right)^{1 / 2} .
\end{aligned}
$$

Because of the spherical symmetry of the problem we can choose the spherical coordinates in a way that $\mu=1$, which leaves

$$
s=\sqrt{r^{2}+r^{\prime 2}-2 r r^{\prime} \mu^{\prime}},
$$

so that Eq. (7) becomes

$$
\begin{aligned}
M(r, \epsilon, t) & =\frac{1}{2 c} \int_{0}^{R} \mathrm{~d} r^{\prime} r^{\prime 2} \int_{-1}^{1} \mathrm{~d} \mu^{\prime} \frac{\rho\left(r^{\prime}, \epsilon, t-\frac{s}{c}\right)}{s^{2}} \\
& =\frac{1}{2 c r} \int_{0}^{R} \mathrm{~d} r^{\prime} r^{\prime} \int_{\left|r-r^{\prime}\right|}^{r+r^{\prime}} \frac{\mathrm{d} s}{s} \rho\left(r^{\prime}, \epsilon, t-\frac{s}{c}\right),
\end{aligned}
$$

where we substituted $s$ from Eq. (9) for the variable $\mu^{\prime}$.

At the source surface $r=R$ the photon intensity is then given by

$$
\begin{aligned}
I(R, \epsilon, t) & =c M(R, \epsilon, t) \\
& =\frac{1}{2 R} \int_{0}^{R} \mathrm{~d} r^{\prime} r^{\prime} \int_{R-r^{\prime}}^{R+r^{\prime}} \frac{\mathrm{d} s}{s} M\left(r^{\prime}, \epsilon, t-\frac{s}{c}\right) .
\end{aligned}
$$

When the surface of the emission cloud is reached, the photons can propagate freely to the observer. The emergent photon intensity therefore equals the surface intensity $I(R, \epsilon, t)$. As noted before, the near isotropy of the synchrotron photon intensity at the edge of the emission knot results from the chaotic magnetic field in the emission knot and multiple Compton scatterings of the generated synchrotron photons after production of the thermal electrons, which allows us to use the diffusion approximation for the spatial transport of synchrotron photons (Sunyaev \& Titarchuk 1980).

If the emission cloud propagates itself with relativistic speed at an angle $\theta$ with respect to the observer, a proper Lorentz transformation of the photon intensity and the photon energy to the observer's frame has to be applied.

The omnidirectional photon production rate is related to the spontaneous photon emission coefficient by

$\rho\left(r, t-\frac{s}{c}\right)=4 \pi j\left(r, t-\frac{s}{c}\right) \exp (-g(x) s / R)$,

where the exponential factor makes use of the escape probability concept of Lightman \& Zdziarski (1987) and Coppi (1992). Here $x=\epsilon /\left(m_{\mathrm{e}} c^{2}\right)$ and

$g(x)=\left[1+\tau_{\mathrm{KN}}(x) f(x)\right]^{-1}$,

$f(x)= \begin{cases}1 & \text { for } \quad x \leq 0.1 \\ (1-x) / 0.9 & \text { for } 0.1<x<1 \\ 0 & \text { for } x \geq 1\end{cases}$

$\tau_{\mathrm{KN}}(x)=\left(\sigma_{\mathrm{T}} R N_{\mathrm{e}}\right) \frac{\sigma_{\mathrm{KN}}(x)}{3 \sigma_{\mathrm{T}}}$,

and

$$
\begin{aligned}
\sigma_{\mathrm{KN}}(x) & =\frac{3 \sigma_{\mathrm{T}}}{8 x}\left[\frac{4}{x}+\frac{2 x(1+x)}{(1+2 x)^{2}}-\frac{2+2 x-x^{2}}{x^{2}} \ln (1+2 x)\right] \\
& \simeq \begin{cases}\sigma_{\mathrm{T}} & \text { for } x \ll 1 \\
\frac{3 \sigma_{\mathrm{T}}}{16 x}[1+2 \ln (2 x)] & \text { for } x \gg 1\end{cases}
\end{aligned}
$$

denotes the total Klein-Nishina cross section.

So instead of a full diffusive description of the synchrotron photon propagation we employ the much simpler escape probability concept here. The escape probability concept represents the fact that, while traversing the distance $s$, a photon has the survival probability $\exp (-g s / R)$ against the dual effects of escaping in a time $R / c$ from the source and at large energies being Compton-scattered to much lower energies. In the relativistic photon energy regime $\epsilon \geq m_{\mathrm{e}} c^{2}$ the large energy shift in a single Compton scattering removes a photon of an energy $\epsilon$ upon scattering. At these photon energies an energy diffusion by 
Comptonization in the source interior no longer occurs (Fabian et al. 1986; Lightman \& Zdziarski 1987). A photon either scatters and appears at a much lower $\epsilon$, or it escapes in a time $R / c$ from the source. Because there are relatively few photons in this relativistic energy region, it is a good approximation to remove these photons in proportion to their probability for scattering and to neglect their reappearance at low energies. According to Lightman \& Zdziarski (1987) the escape probability approach has been well tested with detailed Monte Carlo calculations of photon transport.

\section{Solution of the particle kinetic equation}

\subsection{Eigenfunctions}

The radial diffusion operator of the particle kinetic transport Eq. (4) is of the Sturm-Liouville type so that its eigenfunctions form an orthonormal base in position space. The eigenfunctions are defined by

$$
\frac{c l_{0}}{r^{2}} \frac{\partial}{\partial r}\left(r^{2} \frac{\partial F}{\partial r}\right)+\lambda^{2} F=0
$$

with the boundary conditions of $F(0)$ finite and

$\left[\frac{\mathrm{d} F}{\mathrm{~d} r}+\frac{3}{2 l_{0}} F\right]_{r=R}=0$.

$F(r)$ corresponds to $n(r, \gamma, t)$. The eigenfunction Eq. (17) is solved by

$F(r)=\sum_{k=1}^{\infty} c_{k} F_{k}(r)=\sum_{k=1}^{\infty} c_{k} \frac{\sin \left(\lambda_{k} r\right)}{r}$

in terms of the expansion coefficients $c_{k}$. The solution (19) is finite at $r=0$. According to equation (18) the eigenvalues satisfy the transcendental equation

$\frac{\tan \left(R \lambda_{k}\right)}{R \lambda_{k}}=\frac{1}{1-\frac{3 R}{2 l_{0}}}$.

In the full diffusion limit $l_{0} \ll R$ of many relativistic electron scatterings in the knot, the eigenvalue Eq. (20) can be simplified to

$\tan \left(R \lambda_{k}\right) \simeq-\frac{2 l_{0}}{3} \lambda_{k}$

For $\lambda_{k} l_{0} \ll 1$ Eq. (21) is solved by

$\lambda_{k} \simeq \frac{k \pi}{R}, \quad k=1,2,3, \ldots, k_{\max }$

with $k_{\max } \ll R / l_{0}$. In the case of $\lambda_{k} l_{0} \gg 1$ a solution of Eq. (21) is given by the half-integral multiples of $\pi / R$, but we will see that the course of the functions in the low energy range are dominated by the first eigenvalues, so that the eigenvalues with $k>k_{\max }$ can be neglected. The eigenfunctions $F_{k}(r)$ obey the orthonormality relation

$$
\begin{aligned}
\int_{0}^{R} \mathrm{~d} r r^{2} F_{k}(r) F_{i}(r) & =\left[\int_{0}^{R} \mathrm{~d} r r^{2} F_{k}^{2}(r)\right] \delta_{k i} \\
& =\frac{R \lambda_{k}-\sin \left(\lambda_{k} R\right) \cos \left(\lambda_{k} R\right)}{2 \lambda_{k}} \delta_{k i} .
\end{aligned}
$$

\subsection{Expansion into eigenfunctions}

We can solve the electron kinetic Eq. (4) by expanding the spatial source term and the electron density into the spatial eigenfunctions (19):

$n(r, \gamma, t)=\sum_{k=1}^{\infty} n_{k}(\gamma, t) \frac{\sin \left(\lambda_{k} r\right)}{r}$

and

$q_{2}(r)=\sum_{k=1}^{\infty} b_{k} \frac{\sin \left(\lambda_{k} r\right)}{r}$

where with Eq. (23)

$b_{k}=\frac{2 \lambda_{k}}{R \lambda_{k}-\sin \left(\lambda_{k} R\right) \cos \left(\lambda_{k} R\right)} \int_{0}^{R} \mathrm{~d} r q_{2}(r) r \sin \left(\lambda_{k} r\right)$.

The insertion of the expansions (24) and (25) into the kinetic Eq. (4) and the use of the orthonormality relation (23) yields for the electron expansion functions $n_{k}(\gamma, t)$ the equation

$\frac{\partial n_{k}(\gamma, t)}{\partial t}+\frac{c l_{0} \lambda_{k}^{2}}{3} n_{k}(\gamma, t)-D_{\mathrm{s}} \frac{\partial}{\partial \gamma}\left[\gamma^{2} n_{k}(\gamma, t)\right]=b_{k} q_{1}(\gamma, t)$.

The ansatz

$n_{k}(\gamma, t)=r_{k}(\gamma, t) \mathrm{e}^{-c l_{0} \lambda_{k}^{2} t / 3}$

leads to the equation

$\frac{\partial r_{k}(\gamma, t)}{\partial t}-D_{\mathrm{s}} \frac{\partial}{\partial \gamma}\left[\gamma^{2} r_{k}(\gamma, t)\right]=b_{k} q_{1}(\gamma, t) \mathrm{e}^{c l_{0} \lambda_{k}^{2} t / 3}$,

which for the general injection rate $q_{1}(\gamma, t)$ is solved by

$r_{k}(\gamma, t)=b_{k} \int_{-\infty}^{\infty} \mathrm{d} t^{\prime} \int_{0}^{\infty} \mathrm{d} \gamma^{\prime} q_{1}\left(\gamma^{\prime}, t^{\prime}\right) \mathrm{e}^{c l_{0} \lambda_{k}^{2} t^{\prime} / 3} g_{k}\left(\gamma, \gamma^{\prime}, t, t^{\prime}\right)$.

The equation for the electron Green's function

$\frac{\partial g_{k}(\gamma, t)}{\partial t}-D_{\mathrm{s}} \frac{\partial}{\partial \gamma}\left[\gamma^{2} g_{k}(\gamma, t)\right]=\delta\left(t-t^{\prime}\right) \delta\left(\gamma-\gamma^{\prime}\right)$

has the solution (Kardashev 1962)

$g_{k}\left(\gamma, \gamma^{\prime}, t, t^{\prime}\right)=H\left[t-t^{\prime}\right] \delta\left(\gamma-\frac{\gamma^{\prime}}{1+D_{\mathrm{s}} \gamma^{\prime}\left(t-t^{\prime}\right)}\right)$.

\subsection{Instantaneous injection of monoenergetic ultrarelativistic electrons}

Throughout this work, we consider the instantaneous injection of monoenergetic ultrarelativistic electrons with a density $q_{0}=$ $10^{5} q_{5} \mathrm{~cm}^{-3}$ at the time $t_{0}$ :

$q_{1}(\gamma, t)=q_{0} \delta\left(\gamma-\gamma_{0}\right) \delta\left(t-t_{0}\right)$.

Collecting terms, in this case we obtain for the electron expansion coefficients

$$
\begin{aligned}
n_{k}(\gamma, t)= & q_{0} b_{k} H\left[t-t_{0}\right] \mathrm{e}^{-c l_{0} \lambda_{k}^{2}\left(t-t_{0}\right) / 3} \\
& \times \delta\left(\gamma-\frac{\gamma_{0}}{1+D_{\mathrm{s}} \gamma_{0}\left(t-t_{0}\right)}\right),
\end{aligned}
$$

where $H[x]$ denotes Heaviside's step function. Accordingly, the electron density (24) is given by

$$
\begin{aligned}
n(r, \gamma, t)= & q_{0} H\left[t-t_{0}\right] \delta\left(\gamma-\frac{\gamma_{0}}{1+D_{\mathrm{s}} \gamma_{0}\left(t-t_{0}\right)}\right) \\
& \times \sum_{k=1}^{\infty} b_{k} \frac{\sin \left(\lambda_{k} r\right)}{r} \mathrm{e}^{-c l_{0} \lambda_{k}^{2}\left(t-t_{0}\right) / 3} .
\end{aligned}
$$




\section{Synchrotron radiation}

\subsection{Spontaneous synchrotron emission coefficient}

We first use the electron density distribution (35) to calculate the spontaneous synchrotron emission coefficient $j_{\mathrm{s}}(r, v, t)$, entering the omnidirectional photon production rate distribution (12) and using the monochromatic approximation (Felten \& Morrison 1966) of the synchrotron spectral power in vacuum

$p_{\text {syn }}(\gamma, v)=\frac{c \sigma_{\mathrm{T}} B^{2} \gamma^{2}}{6 \pi} \delta\left(v-v_{\mathrm{s}} \gamma^{2}\right)$

with the characteristic frequency

$v_{\mathrm{s}}=\frac{3 e B}{4 \pi m_{\mathrm{e}} c}=4.2 \times 10^{6} b \mathrm{~Hz}$

for a magnetic field strength of $B=b$ Gauss. $\sigma_{\mathrm{T}}$ denotes the Thomson cross section.

The spontaneous synchrotron emission coefficient is then given by

$$
\begin{aligned}
j_{\mathrm{s}}(r, v, t) & =\frac{1}{4 \pi} \int_{0}^{\infty} \mathrm{d} \gamma n(r, \gamma, t) p_{\text {syn }}(\gamma, v) \\
& =\frac{c \sigma_{\mathrm{T}} B^{2}}{48 \pi^{2} v_{\mathrm{s}}} \sqrt{\frac{v}{v_{\mathrm{s}}}} n\left(r, \gamma=\sqrt{\frac{v}{v_{\mathrm{s}}}}, t\right) .
\end{aligned}
$$

Inserting the electron density distribution (35) we obtain

$$
\begin{aligned}
j_{\mathrm{s}}(r, v, t)= & \frac{c \sigma_{\mathrm{T}} q_{0} B^{2}}{24 \pi^{2}} H\left[t-t_{0}\right] \delta\left(v-\frac{v_{\mathrm{s}} \gamma_{0}^{2}}{\left[1+D_{\mathrm{s}} \gamma_{0}\left(t-t_{0}\right)\right]^{2}}\right) \\
& \times \frac{v}{v_{\mathrm{s}}} \sum_{k=1}^{\infty} b_{k} \frac{\sin \left(\lambda_{k} r\right)}{r} \exp \left[-c l_{0} \lambda_{k}^{2}\left(t-t_{0}\right) / 3\right] .
\end{aligned}
$$

For the corresponding emission coefficient per unit photon energy interval we then derive

$$
\begin{aligned}
j(r, \epsilon, t)= & \frac{1}{h} j_{\mathrm{s}}\left(r, v=\frac{\epsilon}{h}, t\right) \\
= & \frac{c \sigma_{\mathrm{T}} q_{0} B^{2}}{24 \pi^{2}} H\left[t-t_{0}\right] \delta\left(\epsilon-\frac{E_{0}}{\left[1+D_{\mathrm{s}} \gamma_{0}\left(t-t_{0}\right)\right]^{2}}\right) \\
& \times \frac{\epsilon}{h v_{\mathrm{s}}} \sum_{k=1}^{\infty} b_{k} \frac{\sin \left(\lambda_{k} r\right)}{r} \exp \left[-c l_{0} \lambda_{k}^{2}\left(t-t_{0}\right) / 3\right],
\end{aligned}
$$

where we introduce the initial synchrotron photon energy

$$
E_{0}=h v_{\mathrm{s}} \gamma_{0}^{2}=1.74 b \gamma_{7}^{2} \mathrm{MeV}
$$

with the electron Lorentz factor scaling $\gamma_{0}=10^{7} \gamma_{7}$. With Eq. (12) we then find for the omnidirectional synchrotron photon production rate

$$
\begin{aligned}
\rho_{\mathrm{S}}\left(r, t-\frac{s}{c}\right)= & \frac{c \sigma_{\mathrm{T}} q_{0} B^{2}}{6 \pi} \frac{\epsilon}{h v_{\mathrm{S}}} H\left[t-t_{0}-\frac{s}{c}\right] \exp (-g(x) s / R) \\
& \times \delta\left(\epsilon-\frac{E_{0}}{\left[1+D_{\mathrm{s}} \gamma_{0}\left(t-t_{0}-\frac{s}{c}\right)\right]^{2}}\right) \\
& \times \sum_{k=1}^{\infty} b_{k} \frac{\sin \left(\lambda_{k} r\right)}{r} \exp \left[-c l_{0} \lambda_{k}^{2}\left(t-t_{0}-\frac{s}{c}\right) / 3\right]
\end{aligned}
$$

\subsection{Synchrotron photon density inside the emission volume}

For later use we first determine the synchrotron photon density inside the emission volume by inserting Eq. (42) into Eq. (10)

$$
\begin{aligned}
M(r, \epsilon, t)= & \frac{\sigma_{\mathrm{T}} q_{0} B^{2} \gamma_{0}^{2}}{12 \pi r} \frac{\epsilon}{E_{0}} \sum_{k=1}^{\infty} b_{k} \int_{0}^{R} \mathrm{~d} x \sin \left(\lambda_{k} x\right) \\
& \times \int_{|r-x|}^{r+x} \frac{\mathrm{d} s}{s} \exp (-g(x) s / R) H\left[t-t_{0}-\frac{s}{c}\right] \\
& \times \exp \left[-c l_{0} \lambda_{k}^{2}\left(t-t_{0}-\frac{s}{c}\right) / 3\right] \\
& \times \delta\left(\epsilon-\frac{E_{0}}{\left[1+D_{\mathrm{s}} \gamma_{0}\left(t-t_{0}-\frac{s}{c}\right)\right]^{2}}\right) .
\end{aligned}
$$

Substitution of $y=D_{\mathrm{s}} \gamma_{0}\left(t-t_{0}-\frac{s}{c}\right)$ yields

$$
\begin{aligned}
M(r, \epsilon, t)= & \frac{\sigma_{\mathrm{T}} q_{0} B^{2} \gamma_{0}}{12 \pi D_{\mathrm{s}} r} \frac{\epsilon}{E_{0}} \sum_{k=1}^{\infty} b_{k} \int_{0}^{R} \mathrm{~d} x \sin \left(\lambda_{k} x\right) \\
& \times \int_{D_{\mathrm{s}} \gamma_{0}\left(t-t_{0}-\frac{r+x}{c}\right)}^{D_{\mathrm{s}} \gamma_{0}\left(t-t_{0}-\frac{|r-x|}{c}\right)} \frac{\mathrm{d} y H[y]}{t-t_{0}-\frac{y}{D_{\mathrm{s}} \gamma_{0}}} \\
& \times \exp \left(-\frac{g(x) c}{R}\left(t-t_{0}-\frac{y}{D_{\mathrm{s}} \gamma_{0}}\right)-\frac{c l_{0} \lambda_{k}^{2} y}{3 D_{\mathrm{s}} \gamma_{0}}\right) \\
& \times \delta\left(\epsilon-\frac{E_{0}}{[1+y]^{2}}\right) .
\end{aligned}
$$

The $y$-integral is easily evaluated with the help of the $\delta$-function, and we obtain

$$
\begin{aligned}
M(r, \epsilon, t)= & \frac{\sigma_{\mathrm{T}} q_{0} B^{2} \gamma_{0}}{24 \pi D_{\mathrm{s}} r} \frac{H\left[E_{0}-\epsilon\right]}{\left(\epsilon E_{0}\right)^{1 / 2}} \frac{\exp \left(-\frac{g(x) c\left(t-t_{\mathrm{s}}\right)}{R}\right)}{t-t_{\mathrm{s}}} \\
& \times \sum_{k=1}^{\infty} b_{k} \exp \left(-\frac{c l_{0} \lambda_{k}^{2}}{3 D_{\mathrm{s}} \gamma_{0}}\left(\sqrt{\frac{E_{0}}{\epsilon}}-1\right)\right) J(t, r)
\end{aligned}
$$

with the integral

$J(t, r)=\int_{0}^{R} \mathrm{~d} x \sin \left(\lambda_{k} x\right) H\left[x+r-c\left(t-t_{\mathrm{s}}\right)\right] H\left[c\left(t-t_{\mathrm{s}}\right)-|r-x|\right]$,

and we define the photon energy dependent time scale

$$
\begin{aligned}
t_{\mathrm{s}} & \equiv t_{0}+\frac{1}{D_{\mathrm{s}} \gamma_{0}}\left(\sqrt{\frac{E_{0}}{\epsilon}}-1\right) \\
& =t_{0}+\frac{77.3}{b^{2} \gamma_{7}}\left(\sqrt{\frac{1.74 b \gamma_{7}^{2}}{\epsilon(\mathrm{MeV})}}-1\right) \mathrm{s} .
\end{aligned}
$$

With $u=c\left(t-t_{\mathrm{s}}\right)$ the integral (46) yields (Appendix A)

$$
\begin{aligned}
J(t, r)= & \frac{H[u]}{\lambda_{k}}\left(H[r+R-u]\left[\cos \left(\lambda_{k}(r-u)\right)-\cos \left(\lambda_{k} R\right)\right]\right. \\
& \left.+H[R-r-u]\left[\cos \left(\lambda_{k} R\right)-\cos \left(\lambda_{k}(r+u)\right)\right]\right) .
\end{aligned}
$$




\subsection{Emergent synchrotron intensity}

According to Eq. (11) we obtain the emergent synchrotron intensity from Eqs. (45) and (48) as

$$
\begin{aligned}
I(R, \epsilon, t)= & c M(R, \epsilon, t) \\
= & \frac{c \sigma_{\mathrm{T}} q_{0} B^{2} \gamma_{0}}{24 \pi D_{\mathrm{s}} R} \frac{H\left[E_{0}-\epsilon\right]}{\left(\epsilon E_{0}\right)^{1 / 2}} H\left[t-t_{\mathrm{s}}\right] H\left[\frac{2 R}{c}+t_{\mathrm{s}}-t\right] \\
& \times \frac{\exp \left(-\frac{g(x) c\left(t-t_{\mathrm{s}}\right)}{R}\right)}{t-t_{\mathrm{s}}} \\
& \times \sum_{k=1}^{\infty} \frac{b_{k}}{\lambda_{k}} \exp \left(-\frac{c l_{0} \lambda_{k}^{2}}{3 D_{\mathrm{s}} \gamma_{0}}\left(\sqrt{\frac{E_{0}}{\epsilon}}-1\right)\right) \\
& \times\left[\cos \left(\lambda_{k}\left(R-c\left(t-t_{\mathrm{s}}\right)\right)\right)-\cos \left(\lambda_{k} R\right)\right],
\end{aligned}
$$

which gives us the following immediate conclusion of the synchrotron flare duration and flare onset time in the comoving frame of reference:

(1) the total duration of the synchrotron flare at all photon energies $\epsilon$ is $2 R / c$. It has to be noted that the resulting sharp cutoffs (also seen in Fig. 1) are a consequence of the adopted monochromatic approximation of the synchrotron power (36), which ignores the $v^{1 / 3}$ low-frequency dependence of the exact synchrotron power. The monochromatic approximation has been chosen mainly for mathematical convenience, as the exact synchrotron power would imply a much more involved integration over the electron Lorentz factor in Eq. (38). The use of the exact synchrotron power would soften the sharp cut-offs and lead to synchrotron emission of lower intensity for timescales outside the time interval $2 R / c$;

(2) during this energy independent total duration, shorter synchrotron photon energy-dependent time variations are possible, which reflect the influence of the photon retardation and escape like the temporal and spatial dependences of the relativistic electron density distribution through

$$
\begin{aligned}
F_{k}(t)= & \frac{\exp \left(-\frac{g(x) c\left(t-t_{\mathrm{s}}\right)}{R}\right)}{t-t_{\mathrm{s}}} \\
& \times \sum_{k=1}^{\infty} \frac{b_{k}}{\lambda_{k}} \exp \left(-\frac{c l_{0} \lambda_{k}^{2}}{3 D_{\mathrm{s}} \gamma_{0}}\left(\sqrt{\frac{E_{0}}{\epsilon}}-1\right)\right) \\
& \times\left[\cos \left(\lambda_{k}\left(R-c\left(t-t_{\mathrm{s}}\right)\right)\right)-\cos \left(\lambda_{k} R\right)\right] ;
\end{aligned}
$$

(3) the starting time of the flare is energy dependent and given by

$$
\begin{aligned}
t_{\mathrm{s}} & =t_{0}+\frac{1}{D_{\mathrm{s}} \gamma_{0}}\left(\sqrt{\frac{E_{0}}{\epsilon}}-1\right) \\
& =t_{0}+\frac{77.3}{b^{2} \gamma_{7}}\left(\sqrt{\frac{1.74 b \gamma_{7}^{2}}{\epsilon(\mathrm{MeV})}}-1\right) \mathrm{s} .
\end{aligned}
$$

The starting time of the synchrotron flare at photon energy $\epsilon$ is delayed with respect to the injection time of electrons $t_{0}$ by the photon energy dependent time scale $\frac{1}{D_{\mathrm{s}} \gamma_{0}}\left(\sqrt{\frac{E_{0}}{\epsilon}}-1\right)$. This delay reflects the necessary cooling time which relativistic electrons need to radiate at photon energies below $E_{0}$, and its discrete value is also a consequence of using the monochromatic approximation for the synchrotron power. For small photon energies $\epsilon \ll E_{0}$ the intrinsic delay (51) becomes

$t_{\mathrm{s}}-t_{0}=\frac{102}{b^{3 / 2}(\epsilon(\mathrm{MeV}))^{1 / 2}} \mathrm{~s}$.

The onset is delayed further with decreasing photon energy.

\section{Illustrative examples of the emergent synchrotron intensity for different electron injections}

To obtain the full time-dependent development of the synchrotron flare for a given photon energy $\epsilon$, it is necessary to specify in Eq. (26) the spatial electron injection function $q_{2}(r)$, which determines the expansion coefficients $b_{k}$, which enter the synchrotron intensity (49). In our computations we limit the infinite sum over $k$ to a maximum value $k_{\max }$, which is determined by the criterion that the contributions from higher $k$-values provide less than $0.5 \%$ to the total intensity for $t \geq t_{\mathrm{s}}+100 \mathrm{~s}$.

By using the eigenvalues (22), the trigonometric functions in Eq. (49) can be simplified as

$$
\begin{aligned}
I(R, \epsilon, t) \simeq & \frac{c \sigma_{\mathrm{T}} q_{0} B^{2} \gamma_{0}}{12 \pi^{2} D_{\mathrm{s}}} \frac{H\left[t-t_{\mathrm{s}}\right]}{\sqrt{E_{0} \epsilon}} \frac{H\left[E_{0}-\epsilon\right]}{t-t_{\mathrm{s}}} \\
& \times H\left[\frac{2 R}{c}+t_{\mathrm{s}}-t\right] \exp \left(-\frac{g(x) c}{R}\left(t-t_{\mathrm{s}}\right)\right) \\
& \times \sum_{k=1}^{k_{\max }}(-1) \cdot \cos (k \pi) \frac{b_{k}}{k} \\
& \times \exp \left(-\frac{c l_{0} \pi^{2} k^{2}}{3 D_{\mathrm{s}} \gamma_{0} R^{2}}\left(\sqrt{\frac{E_{0}}{\epsilon}}-1\right)\right) \\
& \times \sin ^{2}\left(\frac{k \pi c}{2 R}\left(t-t_{\mathrm{s}}\right)\right)
\end{aligned}
$$

Principally, we have no a-priori knowledge of the exact spatial locations of the electron accelerators in the emission knot, so we chose some illustrative examples of such locations mainly to demonstrate that non-uniform spatial distributions cause short photon energy-dependent synchrotron intensity time variations during the duration of the synchrotron flare. The examples are chosen by mathematical convenience, and some of them may be regarded as artificial and unlikely compared to the unknown true accelerator locations. But these examples serve one important purpose: they indicate that different locations generate different short synchrotron intensity variations. By using future accurate synchrotron light curves one may infer the true accelerator locations, either by forward trial-and-error calculations or by inversion methods.

\subsection{Homogenous spatial electron injection over the whole emission knot}

We first examine the case of a homogeneous electron injection in the whole plasmoid volume, i.e. $q_{2}(r)=1$. From Eq. (26) and the eigenvalues (22) we obtain

$b_{k, 1}=\frac{2}{R} \int_{0}^{R} \mathrm{~d} r r \sin \left(\frac{k \pi}{R} r\right)=\frac{2 R}{k \pi}(-1)^{k+1}$ 


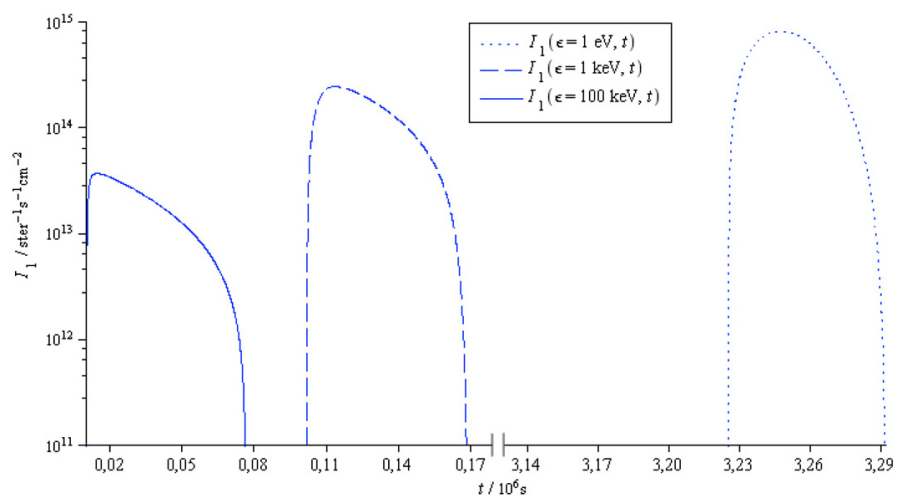

Fig. 1. Intrinsic synchrotron light curves of hard X-rays (solid line), soft $\mathrm{X}$-rays (dashed line) and optical photons (dotted line) resulting from a homogeneous electron injection in the whole plasmoid volume of the size of $R_{15}=1$ and the magnetic field strength of $b=0.1$.

In this case the emitted synchrotron intensity is

$$
\begin{aligned}
I_{1}(\epsilon, t) \simeq & \frac{c \sigma_{\mathrm{T}} q_{0} B^{2} \gamma_{0} R}{6 \pi^{3} D_{\mathrm{s}}} \frac{H\left[t-t_{\mathrm{s}}\right]}{\sqrt{E_{0} \epsilon}} \frac{H\left[E_{0}-\epsilon\right]}{t-t_{\mathrm{s}}} \\
& \times H\left[\frac{2 R}{c}+t_{\mathrm{s}}-t\right] \exp \left(-\frac{g(x) c}{R}\left(t-t_{\mathrm{s}}\right)\right) \\
& \times \sum_{k=1}^{k_{\max }} \frac{1}{k^{2}} \exp \left(-\frac{c l_{0} \pi^{2} k^{2}}{3 D_{\mathrm{s}} \gamma_{0} R^{2}}\left(\sqrt{\frac{E_{0}}{\epsilon}}-1\right)\right) \\
& \times \sin ^{2}\left(\frac{k \pi c}{2 R}\left(t-t_{\mathrm{s}}\right)\right) .
\end{aligned}
$$

For an adopted magnetic field strength of $b=0.1$ we find that for hard $(\epsilon=100 \mathrm{keV}) \mathrm{X}$-rays, soft $(\epsilon=1 \mathrm{keV}) \mathrm{X}$-rays and optical $(\epsilon=1 \mathrm{eV})$ photons, the summation limits are

$k_{\max }(\epsilon=100 \mathrm{keV}) \simeq 61$,

$k_{\max }(\epsilon=1 \mathrm{keV}) \simeq 10$,

$k_{\max }(\epsilon=1 \mathrm{eV}) \simeq 2$.

Figure 1 shows the resulting intrinsic light curves at these three photon energies. In all three cases the total flare duration is $2 R / c=0.67 \times 10^{6} \mathrm{~s}$, as noted in Sect. 4.3 , caused by photon retardation. The flare occurs first at the highest synchrotron photon energies and appears later at lower photon energies, caused by the necessary cooling of relativistic electrons in order to radiate at lower photon energies. All three light curves show that the larger the photon energy, the closer to the onset time $t_{\mathrm{s}}$ is the peak. Moreover, the intensity in the low photon energy range is higher than in the high photon energy range. Thus more intense synchrotron photons are radiated in the optical range $(\epsilon=1 \mathrm{eV})$ some weeks after the electrons were injected into the emission volume $\left(t_{\mathrm{s}}-t_{0} \simeq 37 \mathrm{~d}\right)$.

However, due to the assumed broad homogenous injection condition no variability on shorter time scales than $2 R / c$ is seen in the light curves. Therefore we examine another kind of injection function $q_{2}(r)$ where the electrons are injected homogeneously only in regions close to the boundary of the plasmoid, i.e. $q_{2}(r)=H\left[r-r_{1}\right]$.
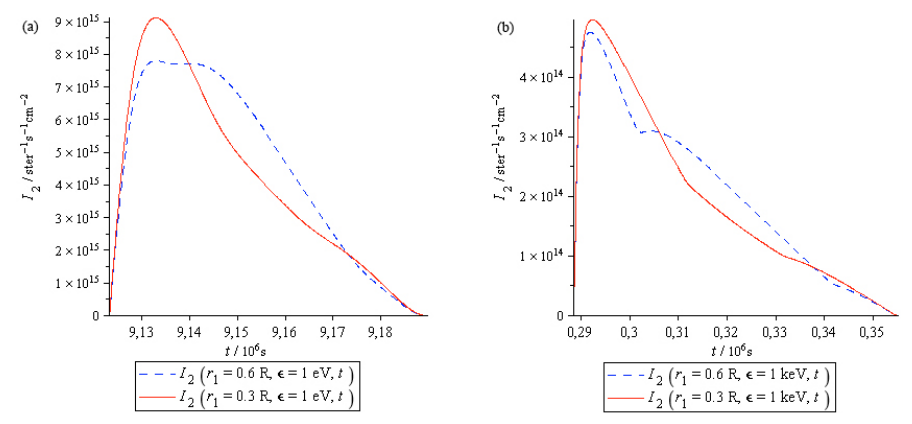

Fig. 2. Temporal flare behaviour of optical photons a) and soft X-rays b) resulting from a homogeneous electron injection over the outer shell $r_{1} \leq r \leq R$ of the plasmoid for $R_{15}=1, b=0.05$ and two values of $r_{1}$.

\subsection{Homogenous spatial electron injection close to the emission knot boundary}

If electrons are injected only at radii between $r_{1} \leq r \leq R$, the spatial injection function is $q_{2}(r)=H\left[r-r_{1}\right]$, which yields for the emission coefficients (26)

$$
\begin{aligned}
b_{k, 2}= & -\frac{2 R}{k^{2} \pi^{2}}\left(k \pi \cos (k \pi)+\sin \left(\frac{k \pi r_{1}}{R}\right)\right. \\
& \left.+\frac{k \pi r_{1}}{R} \cos \left(\frac{k \pi r_{1}}{R}\right)\right) .
\end{aligned}
$$

We then find for the synchrotron intensity

$$
\begin{aligned}
& I_{2}\left(r_{1}, \epsilon, t\right) \simeq \frac{c \sigma_{\mathrm{T}} q_{0} B^{2} \gamma_{0} R}{6 \pi^{4} D_{\mathrm{s}}} \frac{H\left[t-t_{\mathrm{s}}\right]}{\sqrt{E_{0} \epsilon}} \frac{H\left[E_{0}-\epsilon\right]}{t-t_{\mathrm{s}}} \\
& \quad \times H\left[\frac{2 R}{c}+t_{\mathrm{s}}-t\right] \exp \left(-\frac{g(x) c}{R}\left(t-t_{\mathrm{s}}\right)\right) \sum_{k=1}^{k_{\max }} \frac{1}{k^{3}} \\
& \quad \times \sin ^{2}\left(\frac{k \pi c}{2 R}\left(t-t_{\mathrm{s}}\right)\right) \exp \left(-\frac{c l_{0} \pi^{2} k^{2}}{3 D_{\mathrm{s}} \gamma_{0} R^{2}}\left(\sqrt{\frac{E_{0}}{\epsilon}}-1\right)\right) \\
& \quad \times\left(k \pi+\sin \left(k \pi\left(\frac{r_{1}}{R}-1\right)\right)+\frac{k \pi r_{1}}{R} \cos \left(k \pi\left(\frac{r_{1}}{R}-1\right)\right)\right)
\end{aligned}
$$

which is shown in Fig. 2 for $b=0.05$ and two values of $r_{1}$ for soft $(\epsilon=1 \mathrm{keV}) \mathrm{X}$-rays and optical $(\epsilon=1 \mathrm{eV})$ photons. The summation limits in this cases are

$k_{\max }(\epsilon=1 \mathrm{keV}) \simeq 57, \quad k_{\max }(\epsilon=1 \mathrm{eV}) \simeq 11$.

Similar to the first injection scenario we see a steeper rise for the X-rays photons, but to a lower maximum intensity. However, now the light curves in both energy ranges exhibit two maxima in the case of $r_{1} \simeq 0.6 R$, so that variability on shorter time scales than $2 R / c$ can be explained by electron injection only in parts of the emission cloud. The occurrence of such short time variability depends sensitively on the spatial distribution of the injected ultrarelativistic electrons.

\section{Conclusions}

In the previous sections we have investigated an analytic model describing the intrinsic synchrotron intensity emitted by a spherical plasmoid volume in the jet of an AGN. Analytical results for the emergent synchrotron intensity are possible because we have used the monochromatic approximation for the synchrotron power. The synchrotron intensity is given by an infinite sum, reflecting the spatial eigenfunction distribution of the radiating 
electrons over the emission knot. The sum of eigenfunctions results from the time-dependent spatial diffusion of electrons subject to synchrotron energy losses. The radiative transport of the generated synchrotron photons is simplified by using the escape probability concept, which approximates the spatial photon diffusion caused by multiple Compton scatterings off thermal electrons in the knot. With these assumptions we demonstrate that (1) the total duration of the synchrotron flare at all photon energies $\epsilon$ is $2 R / c$, (2) shorter photon energy-dependent synchrotron intensity time variations are possible, which reflect the influence of the photon retardation and escape as well as the temporal and spatial dependences of the relativistic electron density distribution, and (3) the starting time of the synchrotron flare at photon energy $\epsilon$ is delayed with respect to the injection time of electrons $t_{0}$ by the photon energy dependent time scale $\frac{1}{D_{\mathrm{s}} \gamma_{0}}\left(\sqrt{\frac{E_{0}}{\epsilon}}-1\right)$, reflecting the necessary cooling time which relativistic electrons need in order to radiate at photon energies below the initial characteristic synchrotron photon energy $E_{0}$.

The result (2) indicates that time variability different from a single peak in the synchrotron light curves is possible even with a single electron injection if photon retardation and electron propagation effects are properly taken into account. We illustrate this by choosing different spatial distributions of the electron accelerators in the source. By using future accurate synchrotron light curves one thus may infer, either by forward trial-anderror calculations or by inversion methods, the true accelerator locations.

Acknowledgements. We thank the referee for her/his valuable comments. This work was partially supported by the Deutsche Forschungsgemeinschaft through grants RH 35/6-1 and SCHL 201/20-1.

\section{Appendix A: Reduction of the integral (46)}

With the use of $u=c\left(t-t_{\mathrm{s}}\right)$, the integral (46) becomes

$J(t, r)=\int_{0}^{R} \mathrm{~d} r^{\prime} \sin \left(\lambda_{k} r^{\prime}\right) H\left[r^{\prime}+r-u\right] H\left[u-\left|r-r^{\prime}\right|\right]$.

Because of the Heaviside functions, the integral has to be examined in three different ranges, as seen in Fig. A.1, which yields

$$
\begin{aligned}
J(t, r)= & H[u]\left(H[r-u] \int_{r-u}^{R} \mathrm{~d} r^{\prime} \sin \left(\lambda_{k} r^{\prime}\right)+H[u-r]\right. \\
& \times H[r+R-u] \int_{-r+u}^{R} \mathrm{~d} r^{\prime} \sin \left(\lambda_{k} r^{\prime}\right) \\
& \left.-H[R-u-r] \int_{u+r}^{R} \mathrm{~d} r^{\prime} \sin \left(\lambda_{k} r^{\prime}\right)\right) .
\end{aligned}
$$

As the cosine is an even function, the first two integrals could be merged to one in the range of $0 \leq u \leq r+R$, leading to

$$
\begin{aligned}
J(t, r)= & \frac{H[u]}{\lambda_{k}}\left(H[r+R-u]\left(\cos \left(\lambda_{k}(r-u)\right)-\cos \left(\lambda_{k} R\right)\right)\right. \\
& \left.+H[R-u-r]\left(\cos \left(\lambda_{k} R\right)-\cos \left(\lambda_{k}(r+u)\right)\right)\right) .
\end{aligned}
$$

\section{Appendix B: invariance relations}

The transformation formulae regarding photon intensities result from the Lorentz invariance of the photon phase space density (Begelman et al. 1984)

$\mathcal{P}=\frac{\mathrm{d} N}{\mathrm{~d} \mathcal{V}}=\mathcal{P}^{*}$

Page 8 of 8

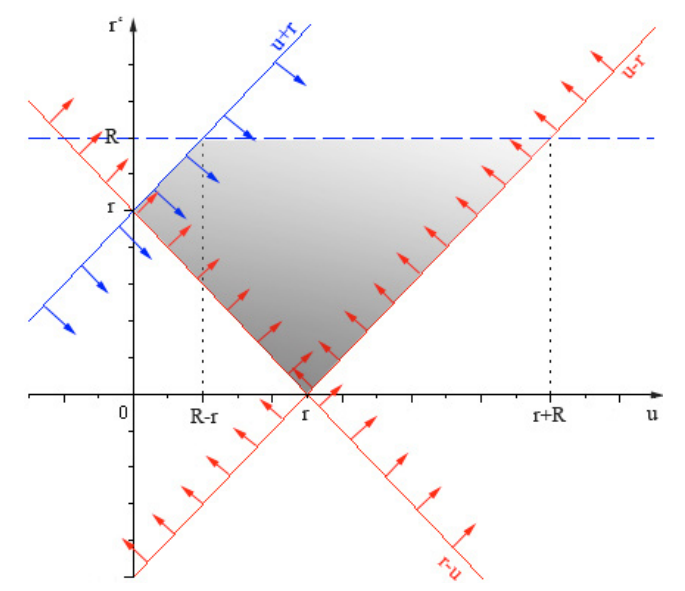

Fig. A.1. The grey area illustrates the range of integration. The lower limit $r^{\prime}=u-r$ is given by $H\left[r^{\prime}+r-u\right] . H\left[u-\left|r-r^{\prime}\right|\right]$ gives the lower $\operatorname{limt} r^{\prime}=r-u$, as well as the upper limit $r^{\prime}=u+r$.

With the photon energy-momentum relation $\epsilon=p c$ and spherical momentum coordinates (i.e. $\mathrm{d}^{3} p=p^{2} \mathrm{~d} p \mathrm{~d} \Omega$ ) we obtain

$\mathcal{P}=\frac{c^{3} \mathrm{~d} N}{\epsilon^{2} \mathrm{~d} \epsilon \mathrm{d} \Omega \mathrm{d}^{3} x}$.

For the differential photon density $M(r, \epsilon, t)=\frac{\mathrm{d} N}{\mathrm{~d} \Omega \mathrm{d}^{3} x}$ this gives the invariant

$\frac{M}{\epsilon^{2} \mathrm{~d} \epsilon}=\frac{M^{*}}{\epsilon^{* 2} \mathrm{~d} \epsilon^{*}}$

so that the intensity $I=c M$ relation is

$I^{*}\left(R, \epsilon^{*}, t^{*}\right)=\delta^{3} I\left(R, \epsilon=\frac{\epsilon^{*}}{\delta}, t=t^{*} \delta\right)$.

Times and photon energies transform as

$t^{*}=\delta^{-1} t$ and $t_{\mathrm{s}}^{*}=\delta^{-1} t_{\mathrm{s}}$,

$\epsilon^{*}=\delta \epsilon \quad$ and $\quad E_{0}^{*}=\delta E_{0}$.

\section{References}

Aharonian, F. A., et al. (HESS Collaboration) 2007, ApJ, 664, L7

Albert, J., Aliu, E., Anderhub, H., et al. (MAGIC Collaboration) 2007, ApJ, 663, 125

Begelman, M. C., Blandford, R. D., \& Rees, M. J. 1984, Rev. Mod. Phys., 56, 2 Coppi, P. S. 1992, MNRAS, 258, 657

Crusius, A., \& Schlickeiser, R. 1986, A\&A, 164, 16

Crusius, A., \& Schlickeiser, R. 1988, A\&A, 196, 327

Fabian, A. C., Blandford, R. D., Guilbert, P. W., Phinney, E. S., \& Cuellar, L. 1986, MNRAS, 221, 931

Felten, J. E., \& Morrison, P. 1966, ApJ, 146, 686

Gould, R. J. 1979, A\&A, 76, 306

Lightman, A. P., \& Zdziarski, A. A. 1987, ApJ, 319, 643

Piner, B. G., \& Edwards, P. G. 2004, ApJ, 600, 115

Schlickeiser, R. 1996, Space Sci. Rev., 75, 299

Schlickeiser, R. 2009, MNRAS, 398, 1483

Schlickeiser, R., \& Lerche, I. 2007, A\&A, 476, 1

Schlickeiser, R., \& Röken, C. 2008, A\&A, 477, 701 (SR)

Schlickeiser, R., Vainio, R., Böttcher, M., et al. 2002, A\&A, 393, 69

Stockem, A., \& Schlickeiser, R. 2008, ApJ, 680, 816

Sunyaev, R. A., \& Titarchuk, L. G. 1980, A\&A, 86, 121

Sunyaev, R. A., \& Titarchuk, L. G. 1985, A\&A, 143, 374

Turner, M., et al. 2003, Connecting Quarks with the Cosmos (Washington: The National Academic Press)

Woo, J.-H., Urry, C. M., van der Marel, R. P., Lira, P., \& Maza, J., et al. 2005, ApJ, 631, 762 